\title{
Algorithms with strong convergence for the split common solution of the feasibility problem and fixed point problem
}

Yonghong Yao ${ }^{1}$, Ravi P Agarwal ${ }^{2,3}$, Mihai Postolache ${ }^{4 *}$ and Yeong-Cheng Liou ${ }^{5}$

\section{"Correspondence:}

mihai@mathem.pub.ro

${ }^{4}$ Faculty of Applied Sciences,

University 'Politehnica' of Bucharest,

Splaiul Independentei 313,

Bucharest, 060042, Romania

Full list of author information is

available at the end of the article

\begin{abstract}
The purpose of this paper is to study the split feasibility problem and fixed point problem involved in the pseudocontractive mappings. We construct an iterative algorithm and prove its strong convergence.

MSC: 47J25; 47H09; 65J15; 90C25
\end{abstract}

Keywords: split feasibility problem; fixed point problem; pseudocontractive mapping

\section{Introduction}

Let $H_{1}$ and $H_{2}$ be two real Hilbert spaces and let $C \subset H_{1}$ and $Q \subset H_{2}$ be two nonempty, closed, and convex sets. Let $A: H_{1} \rightarrow H_{2}$ be a bounded linear operator with its adjoint $A^{*}$. Let $S: H_{2} \rightarrow H_{2}$ and $T: H_{1} \rightarrow H_{1}$ be two nonlinear mappings.

The purpose of this paper is to study the following split feasibility problem and fixed point problem:

$$
\text { Find } x^{*} \in C \cap \operatorname{Fix}(T) \text { such that } A x^{*} \in Q \cap \operatorname{Fix}(S) \text {. }
$$

Special cases:

(i) Finding a point $x^{*}$ which satisfies

$$
x^{*} \in C \text { and } A x^{*} \in Q \text {. }
$$

This problem, referred to as the split feasibility problem, was introduced by Censor and Elfving [1], modeling phase retrieval and other image restoration problems, and further studied by many researchers; see, for instance, [2-7].

(ii) Find a point $x^{*}$ with the property

$$
x^{*} \in \operatorname{Fix}(T) \text { and } A x^{*} \in \operatorname{Fix}(S) .
$$

This problem, referred to as the split common fixed point problem, was first introduced by Censor and Segal [8].

Next, we recall some existing algorithms for solving (1.1)-(1.3) in the literature.

O2014 Yao et al.: licensee Springer. This is an Open Access article distributed under the terms of the Creative Commons Attribution License (http://creativecommons.org/licenses/by/2.0), which permits unrestricted use, distribution, and reproduction in any medium, provided the original work is properly cited. 
In order to solve (1.2), Censor and Elfving [1] introduced the following algorithm:

$$
x_{n+1}=A^{-1} P_{Q}\left(P_{A(C)}\left(A x_{n}\right)\right), \quad n \in \mathbb{N},
$$

where $C$ and $Q$ are closed and convex sets in $\mathbb{R}^{n}, A$ is a full rank $n \times n$ matrix and $A(C)=$ $\left\{y \in \mathbb{R}^{n} \mid y=A x, x \in C\right\}$.

Now (1.4) is not popular because it involves the computation of the inverse $A^{-1}$.

A more popular algorithm that solves (1.4) seems to be the $C Q$ algorithm presented by Byrne $[5,7]$ :

$$
x_{n+1}=P_{C}\left(x_{n}-\tau A^{*}\left(I-P_{Q}\right) A x_{n}\right), \quad n \in \mathbb{N},
$$

where $\tau \in\left(0, \frac{2}{L}\right)$, with $L$ being the largest eigenvalue of the matrix $A^{*} A$.

Note that $x^{*}$ solves (1.2) if and only if $x^{*}$ solves the fixed point equation

$$
x^{*}=P_{C}\left(I-\lambda A^{*}\left(I-P_{Q}\right) A\right) x^{*} .
$$

The above equivalence relation (1.6) reminds us to use fixed point method to solve (1.2) Many authors have given a continuation of the study on the CQ algorithm and its variant form. For related work, please refer to [9-16]. Especially, the following regularized method was presented by $\mathrm{Xu}[6]$ :

$$
x_{n+1}=P_{C}\left(\left(1-\alpha_{n} \gamma_{n}\right) x_{n}-\gamma_{n} A^{*}\left(I-P_{Q}\right) A x_{n}\right), \quad n \in \mathbb{N} .
$$

It should be pointed out that (1.7) can be used to find the minimum norm solution of (1.2).

For solving (1.3), Censor and Segal [8] invented an algorithm which generates a sequence $\left\{x_{n}\right\}$ according to the iterative procedure:

$$
x_{n+1}=T\left(x_{n}-\gamma A^{*}(I-S) A x_{n}\right), \quad n \in \mathbb{N} .
$$

Note that (1.8) is more general than (1.5). Some further generations of this algorithm were studied by Moudafi [17] and Wang and Xu [18] and others; see, for example, [19-22].

Motivated by the results in this direction, the purpose of this paper is to study the split feasibility problem and the fixed point problem involved in the pseudocontractive mappings. We construct an iterative algorithm and prove its strong convergence.

\section{Preliminaries}

Let $H$ be a real Hilbert space with inner product $\langle\cdot, \cdot\rangle$ and norm $\|\cdot\|$, respectively. Let $C$ be a nonempty, closed, and convex subset of $H$.

Recall that a mapping $T: C \rightarrow C$ is called pseudocontractive if

$$
\langle T x-T y, x-y\rangle \leq\|x-y\|^{2}
$$

for all $x, y \in C$. It is well known that $T$ is pseudocontractive if and only if

$$
\|T x-T y\|^{2} \leq\|x-y\|^{2}+\|(I-T) x-(I-T) y\|^{2}
$$


for all $x, y \in C$. A mapping $T: C \rightarrow C$ is called L-Lipschitzian if there exists $L>0$ such that

$$
\|T x-T y\| \leq L\|x-y\|
$$

for all $x, y \in C$. If $L=1$, we call $T$ nonexpansive.

We will use Fix $(T)$ to denote the set of fixed points of $T$, that is,

$$
\operatorname{Fix}(T)=\{x \in C: x=T x\} .
$$

We know that the metric projection $P_{C}: H \rightarrow C$ satisfies

$$
\left\|x-P_{C}(x)\right\|=\inf \{\|x-y\|: y \in C\} .
$$

It is well known that the metric projection $P_{C}: H \rightarrow C$ is firmly nonexpansive, that is,

$$
\begin{aligned}
& \left\langle x-y, P_{C}(x)-P_{C}(y)\right\rangle \geq\left\|P_{C}(x)-P_{C}(y)\right\|^{2} \\
& \quad \Leftrightarrow \quad\left\|P_{C}(x)-P_{C}(y)\right\|^{2} \leq\|x-y\|^{2}-\left\|\left(I-P_{C}\right) x-\left(I-P_{C}\right) y\right\|^{2}
\end{aligned}
$$

for all $x, y \in H$.

For all $x, y \in H$, the following conclusions hold:

$$
\begin{aligned}
& \|t x+(1-t) y\|^{2}=t\|x\|^{2}+(1-t)\|y\|^{2}-t(1-t)\|x-y\|^{2}, \quad t \in[0,1], \\
& \|x+y\|^{2}=\|x\|^{2}+2\langle x, y\rangle+\|y\|^{2},
\end{aligned}
$$

and

$$
\|x+y\|^{2} \leq\|x\|^{2}+2\langle y, x+y\rangle .
$$

Lemma 2.1 ([23]) Let H be a real Hilbert space, $C$ a closed convex subset of H. Let $T: C \rightarrow$ $C$ be a continuous pseudocontractive mapping. Then

(i) $\operatorname{Fix}(T)$ is a closed convex subset of $C$,

(ii) $(I-T)$ is demiclosed at zero.

Lemma 2.2 ([24]) Assume that $\left\{a_{n}\right\}$ is a sequence of nonnegative real numbers such that

$$
a_{n+1} \leq\left(1-\gamma_{n}\right) a_{n}+\delta_{n}, \quad n \in \mathbb{N},
$$

where $\left\{\gamma_{n}\right\}$ is a sequence in $(0,1)$ and $\left\{\delta_{n}\right\}$ is a sequence such that

(1) $\sum_{n=1}^{\infty} \gamma_{n}=\infty$;

(2) $\lim \sup _{n \rightarrow \infty} \frac{\delta_{n}}{\gamma_{n}} \leq 0$ or $\sum_{n=1}^{\infty}\left|\delta_{n}\right|<\infty$.

Then $\lim _{n \rightarrow \infty} a_{n}=0$.

Lemma 2.3 ([25]) Let $\left\{w_{n}\right\}$ be a sequence of real numbers. Assume $\left\{w_{n}\right\}$ does not decrease at infinity, that is, there exists at least a subsequence $\left\{w_{n_{k}}\right\}$ of $\left\{w_{n}\right\}$ such that $w_{n_{k}} \leq w_{n_{k}+1}$ for all $k \geq 0$. For every $n \geq N_{0}$, define an integer sequence $\{\tau(n)\}$ as

$$
\tau(n)=\max \left\{i \leq n: w_{n_{i}}<w_{n_{i}+1}\right\} .
$$


Then $\tau(n) \rightarrow \infty$ as $n \rightarrow \infty$ and for all $n \geq N_{0}$

$$
\max \left\{w_{\tau(n)}, w_{n}\right\} \leq w_{\tau(n)+1} .
$$

\section{Main results}

Let $H_{1}$ and $H_{2}$ be two real Hilbert spaces and let $C \subset H_{1}$ and $Q \subset H_{2}$ be two nonempty closed convex sets. Let $A: H_{1} \rightarrow H_{2}$ be a bounded linear operator with its adjoint $A^{*}$. Let $S: H_{2} \rightarrow H_{2}$ nonexpansive mapping and let $T: H_{1} \rightarrow H_{1}$ be an $L$-Lipschitzian pseudocontractive mapping with $L>1$.

We use $\Gamma$ to denote the set of solutions of (1.1), that is,

$$
\Gamma=\left\{x^{*} \mid x^{*} \in C \cap \operatorname{Fix}(T), A x^{*} \in Q \cap \operatorname{Fix}(S)\right\} .
$$

In the sequel, we assume $\Gamma \neq \emptyset$.

Now, we present our algorithm for finding $x^{*} \in \Gamma$.

Algorithm 3.1 For fixed $u \in H_{1}$ and $x_{0} \in H_{1}$ arbitrarily, let $\left\{x_{n}\right\}$ be a sequence defined by

$$
\left\{\begin{array}{l}
u_{n}=P_{C}\left[\alpha_{n} u+\left(1-\alpha_{n}\right)\left(x_{n}-\delta A^{*}\left(I-S P_{Q}\right) A x_{n}\right)\right], \\
x_{n+1}=\left(1-\beta_{n}\right) u_{n}+\beta_{n} T\left(\left(1-\gamma_{n}\right) u_{n}+\gamma_{n} T u_{n}\right), \quad n \in \mathbb{N},
\end{array}\right.
$$

where $\left\{\alpha_{n}\right\}_{n \in \mathbb{N}},\left\{\beta_{n}\right\}_{n \in \mathbb{N}}$, and $\left\{\gamma_{n}\right\}_{n \in \mathbb{N}}$ are three real number sequences in $(0,1)$ and $\delta$ is a constant in $\left(0, \frac{1}{\|A\|^{2}}\right)$.

Theorem 3.2 Assume the following conditions are satisfied:

(C1) $\lim _{n \rightarrow \infty} \alpha_{n}=0$;

(C2) $\sum_{n=1}^{\infty} \alpha_{n}=\infty$;

(C3) $0<a<\beta_{n}<c<\gamma_{n}<b<\frac{1}{\sqrt{1+L^{2}}+1}$.

Then the sequence $\left\{x_{n}\right\}$ generated by algorithm (3.1) converges strongly to the point $x^{*}$, given by $x^{*}=P_{\Gamma}(u)$.

Proof Set $x^{*}=P_{\Gamma}(u)$. Then we have $x^{*} \in C \cap \operatorname{Fix}(T)$ and $A x^{*} \in Q \cap \operatorname{Fix}(S)$. Set $z_{n}=P_{Q} A x_{n}$ and $y_{n}=\alpha_{n} u+\left(1-\alpha_{n}\right)\left(x_{n}-\delta A^{*}\left(I-S P_{Q}\right) A x_{n}\right)$ for all $n \in \mathbb{N}$. Thus $u_{n}=P_{C} y_{n}$ for all $n \in \mathbb{N}$.

Since $P_{C}$ and $P_{Q}$ are nonexpansive, we have

$$
\left\|z_{n}-A x^{*}\right\|=\left\|P_{Q} A x_{n}-P_{Q} A x^{*}\right\| \leq\left\|A x_{n}-A x^{*}\right\|
$$

and

$$
\left\|u_{n}-x^{*}\right\|=\left\|P_{C} y_{n}-P_{C} x^{*}\right\| \leq\left\|y_{n}-x^{*}\right\| .
$$

By (2.2), we get

$$
\begin{aligned}
\left\|S z_{n}-A x^{*}\right\|^{2} & =\left\|S P_{Q} A x_{n}-S P_{Q} A x^{*}\right\|^{2} \\
& \leq\left\|P_{Q} A x_{n}-P_{Q} A x^{*}\right\|^{2} \\
& \leq\left\|A x_{n}-A x^{*}\right\|^{2}-\left\|z_{n}-A x_{n}\right\|^{2} .
\end{aligned}
$$


From (2.1), we have

$$
\left\|T u_{n}-x^{*}\right\|^{2} \leq\left\|u_{n}-x^{*}\right\|^{2}+\left\|T u_{n}-u_{n}\right\|^{2}
$$

and

$$
\begin{aligned}
\| T & \left(\left(1-\gamma_{n}\right) u_{n}+\gamma_{n} T u_{n}\right)-x^{*} \|^{2} \\
\leq & \left\|\left(1-\gamma_{n}\right)\left(u_{n}-x^{*}\right)+\gamma_{n}\left(T u_{n}-x^{*}\right)\right\|^{2} \\
& \quad+\left\|\left(1-\gamma_{n}\right) u_{n}+\gamma_{n} T u_{n}-T\left(\left(1-\gamma_{n}\right) u_{n}+\gamma_{n} T u_{n}\right)\right\|^{2}
\end{aligned}
$$

Applying equality (2.3), we have

$$
\begin{aligned}
\left\|\left(1-\gamma_{n}\right) u_{n}+\gamma_{n} T u_{n}-T\left(\left(1-\gamma_{n}\right) u_{n}+\gamma_{n} T u_{n}\right)\right\|^{2} \\
=\left\|\left(1-\gamma_{n}\right)\left(u_{n}-T\left(\left(1-\gamma_{n}\right) u_{n}+\gamma_{n} T u_{n}\right)\right)+\gamma_{n}\left(T u_{n}-T\left(\left(1-\gamma_{n}\right) u_{n}+\gamma_{n} T u_{n}\right)\right)\right\|^{2} \\
=\left(1-\gamma_{n}\right)\left\|u_{n}-T\left(\left(1-\gamma_{n}\right) u_{n}+\gamma_{n} T u_{n}\right)\right\|^{2}+\gamma_{n}\left\|T u_{n}-T\left(\left(1-\gamma_{n}\right) u_{n}+\gamma_{n} T u_{n}\right)\right\|^{2} \\
\quad-\gamma_{n}\left(1-\gamma_{n}\right)\left\|u_{n}-T u_{n}\right\|^{2} .
\end{aligned}
$$

Since $T$ is $L$-Lipschitzian and $u_{n}-\left(\left(1-\gamma_{n}\right) u_{n}+\gamma_{n} T u_{n}\right)=\gamma_{n}\left(u_{n}-T u_{n}\right)$, by (3.7), we get

$$
\begin{aligned}
&\left\|\left(1-\gamma_{n}\right) u_{n}+\gamma_{n} T u_{n}-T\left(\left(1-\gamma_{n}\right) u_{n}+\gamma_{n} T u_{n}\right)\right\|^{2} \\
& \leq\left(1-\gamma_{n}\right)\left\|u_{n}-T\left(\left(1-\gamma_{n}\right) u_{n}+\gamma_{n} T u_{n}\right)\right\|^{2}+\gamma_{n}^{3} L^{2}\left\|u_{n}-T u_{n}\right\|^{2} \\
&-\gamma_{n}\left(1-\gamma_{n}\right)\left\|u_{n}-T u_{n}\right\|^{2} \\
&=\left(1-\gamma_{n}\right)\left\|u_{n}-T\left(\left(1-\gamma_{n}\right) u_{n}+\gamma_{n} T u_{n}\right)\right\|^{2}+\left(\gamma_{n}^{3} L^{2}+\gamma_{n}^{2}-\gamma_{n}\right)\left\|u_{n}-T u_{n}\right\|^{2} .
\end{aligned}
$$

By (2.3) and (3.5), we have

$$
\begin{aligned}
\|( & \left.-\gamma_{n}\right)\left(u_{n}-x^{*}\right)+\gamma_{n}\left(T u_{n}-x^{*}\right) \|^{2} \\
= & \left(1-\gamma_{n}\right)\left\|u_{n}-x^{*}\right\|^{2}+\gamma_{n}\left\|T u_{n}-x^{*}\right\|^{2}-\gamma_{n}\left(1-\gamma_{n}\right)\left\|u_{n}-T u_{n}\right\|^{2} \\
\leq & \left(1-\gamma_{n}\right)\left\|u_{n}-x^{*}\right\|^{2}+\gamma_{n}\left(\left\|u_{n}-x^{*}\right\|^{2}+\left\|u_{n}-T u_{n}\right\|^{2}\right) \\
& \quad-\gamma_{n}\left(1-\gamma_{n}\right)\left\|u_{n}-T u_{n}\right\|^{2} \\
= & \left\|u_{n}-x^{*}\right\|^{2}+\gamma_{n}^{2}\left\|u_{n}-T u_{n}\right\|^{2} .
\end{aligned}
$$

From (3.6), (3.8), and (3.9), we deduce

$$
\begin{aligned}
\| T & \left(\left(1-\gamma_{n}\right) u_{n}+\gamma_{n} T u_{n}\right)-x^{*} \|^{2} \\
\leq & \left\|u_{n}-x^{*}\right\|^{2}+\left(1-\gamma_{n}\right)\left\|u_{n}-T\left(\left(1-\gamma_{n}\right) u_{n}+\gamma_{n} T u_{n}\right)\right\|^{2} \\
& \quad-\gamma_{n}\left(1-2 \gamma_{n}-\gamma_{n}^{2} L^{2}\right)\left\|u_{n}-T u_{n}\right\|^{2} .
\end{aligned}
$$

Since $\gamma_{n}<b<\frac{1}{\sqrt{1+L^{2}}+1}$, we derive that

$$
1-2 \gamma_{n}-\gamma_{n}^{2} L^{2}>0, \quad \forall n \in \mathbb{N} \text {. }
$$


This together with (3.10) implies that

$$
\begin{aligned}
& \left\|T\left(\left(1-\gamma_{n}\right) u_{n}+\gamma_{n} T u_{n}\right)-x^{*}\right\|^{2} \\
& \quad \leq\left\|u_{n}-x^{*}\right\|^{2}+\left(1-\gamma_{n}\right)\left\|u_{n}-T\left(\left(1-\gamma_{n}\right) u_{n}+\gamma_{n} T u_{n}\right)\right\|^{2} .
\end{aligned}
$$

By (2.3), (3.1), (3.11), and (C3), we have

$$
\begin{aligned}
\left\|x_{n+1}-x^{*}\right\|^{2}= & \left\|\left(1-\beta_{n}\right) u_{n}+\beta_{n} T\left(\left(1-\gamma_{n}\right) u_{n}+\gamma_{n} T u_{n}\right)-x^{*}\right\|^{2} \\
= & \left(1-\beta_{n}\right)\left\|u_{n}-x^{*}\right\|^{2}+\beta_{n}\left\|T\left(\left(1-\gamma_{n}\right) u_{n}+\gamma_{n} T u_{n}\right)-x^{*}\right\|^{2} \\
& -\beta_{n}\left(1-\beta_{n}\right)\left\|u_{n}-T\left(\left(1-\gamma_{n}\right) u_{n}+\gamma_{n} T u_{n}\right)\right\|^{2} \\
\leq & \left\|u_{n}-x^{*}\right\|^{2}-\beta_{n}\left(\gamma_{n}-\beta_{n}\right)\left\|T\left(\left(1-\gamma_{n}\right) u_{n}+\gamma_{n} T u_{n}\right)-x^{*}\right\|^{2} \\
\leq & \left\|u_{n}-x^{*}\right\|^{2} .
\end{aligned}
$$

By the convexity of the norm and by using (2.4), we get

$$
\begin{aligned}
\left\|y_{n}-x^{*}\right\|^{2}= & \left\|\alpha_{n}\left(u-x^{*}\right)+\left(1-\alpha_{n}\right)\left(x_{n}-x^{*}+\delta A^{*}\left(S z_{n}-A x_{n}\right)\right)\right\|^{2} \\
\leq & \left(1-\alpha_{n}\right)\left\|\left(x_{n}-x^{*}+\delta A^{*}\left(S z_{n}-A x_{n}\right)\right)\right\|^{2}+\alpha_{n}\left\|u-x^{*}\right\|^{2} \\
= & \left(1-\alpha_{n}\right)\left[\left\|x_{n}-x^{*}\right\|+\delta^{2}\left\|A^{*}\left(S z_{n}-A x_{n}\right)\right\|^{2}\right. \\
& \left.+2 \delta\left(x_{n}-x^{*}, A^{*}\left(S z_{n}-A x_{n}\right)\right\rangle\right]+\alpha_{n}\left\|u-x^{*}\right\|^{2} .
\end{aligned}
$$

Since $A$ is a linear operator with its adjoint $A^{*}$, we have

$$
\begin{aligned}
\left\langle x_{n}\right. & \left.-x^{*}, A^{*}\left(S z_{n}-A x_{n}\right)\right\rangle \\
& =\left\langle A\left(x_{n}-x^{*}\right), S z_{n}-A x_{n}\right\rangle \\
& =\left\langle A x_{n}-A x^{*}+S z_{n}-A x_{n}-\left(S z_{n}-A x_{n}\right), S z_{n}-A x_{n}\right\rangle \\
& =\left\langle S z_{n}-A x^{*}, S z_{n}-A x_{n}\right\rangle-\left\|S z_{n}-A x_{n}\right\|^{2} .
\end{aligned}
$$

Again using (2.4), we obtain

$$
\left\langle S z_{n}-A x^{*}, S z_{n}-A x_{n}\right\rangle=\frac{1}{2}\left(\left\|S z_{n}-A x^{*}\right\|^{2}+\left\|S z_{n}-A x_{n}\right\|^{2}-\left\|A x_{n}-A x^{*}\right\|^{2}\right) .
$$

From (3.4), (3.14), and (3.15), we get

$$
\begin{aligned}
\left\langle x_{n}-x^{*}, A^{*}\left(S z_{n}-A x_{n}\right)\right\rangle= & \frac{1}{2}\left(\left\|S z_{n}-A x^{*}\right\|^{2}+\left\|S z_{n}-A x_{n}\right\|^{2}-\left\|A x_{n}-A x^{*}\right\|^{2}\right) \\
& -\left\|S z_{n}-A x_{n}\right\|^{2} \\
\leq & \frac{1}{2}\left(\left\|A x_{n}-A x^{*}\right\|^{2}-\left\|z_{n}-A x_{n}\right\|^{2}+\left\|S z_{n}-A x_{n}\right\|^{2}\right. \\
& \left.-\left\|A x_{n}-A x^{*}\right\|^{2}\right)-\left\|S z_{n}-A x_{n}\right\|^{2} \\
= & -\frac{1}{2}\left\|z_{n}-A x_{n}\right\|^{2}-\frac{1}{2}\left\|S z_{n}-A x_{n}\right\|^{2} .
\end{aligned}
$$


Substituting (3.16) into (3.13) we deduce

$$
\begin{aligned}
\left\|y_{n}-x^{*}\right\|^{2} \leq & \left(1-\alpha_{n}\right)\left[\left\|x_{n}-x^{*}\right\|^{2}+\delta^{2}\|A\|^{2}\left\|S z_{n}-A x_{n}\right\|^{2}\right. \\
& \left.+2 \delta\left(-\frac{1}{2}\left\|z_{n}-A x_{n}\right\|^{2}-\frac{1}{2}\left\|S z_{n}-A x_{n}\right\|^{2}\right)\right]+\alpha_{n}\left\|u-x^{*}\right\|^{2} \\
= & \left(1-\alpha_{n}\right)\left[\left\|x_{n}-x^{*}\right\|^{2}+\left(\delta^{2}\|A\|^{2}-\delta\right)\left\|S z_{n}-A x_{n}\right\|^{2}\right. \\
& \left.-\delta\left\|z_{n}-A x_{n}\right\|^{2}\right]+\alpha_{n}\left\|u-x^{*}\right\|^{2} \\
\leq & \left(1-\alpha_{n}\right)\left\|x_{n}-x^{*}\right\|^{2}+\alpha_{n}\left\|u-x^{*}\right\|^{2} .
\end{aligned}
$$

From (3.3), (3.12), and (3.17), we get

$$
\begin{aligned}
\left\|x_{n+1}-x^{*}\right\|^{2} & \leq\left\|y_{n}-x^{*}\right\|^{2} \\
& \leq\left(1-\alpha_{n}\right)\left\|x_{n}-x^{*}\right\|^{2}+\alpha_{n}\left\|u-x^{*}\right\|^{2} \\
& \leq \max \left\{\left\|x_{n}-x^{*}\right\|^{2},\left\|u-x^{*}\right\|^{2}\right\} .
\end{aligned}
$$

The boundedness of the sequence $\left\{x_{n}\right\}$ yields our result.

Using the firmly nonexpansivenessity of $P_{C}(2.2)$, we have

$$
\begin{aligned}
\left\|u_{n}-x^{*}\right\|^{2} & =\left\|P_{C} y_{n}-x^{*}\right\|^{2} \leq\left\|y_{n}-x^{*}\right\|^{2}-\left\|P_{C} y_{n}-y_{n}\right\|^{2} \\
& =\left\|y_{n}-x^{*}\right\|^{2}-\left\|u_{n}-y_{n}\right\|^{2} .
\end{aligned}
$$

Thus

$$
\begin{aligned}
\left\|x_{n+1}-x^{*}\right\|^{2} & \leq\left\|u_{n}-x^{*}\right\|^{2} \\
& \leq\left\|y_{n}-x^{*}\right\|^{2}-\left\|u_{n}-y_{n}\right\|^{2} \\
& \leq\left(1-\alpha_{n}\right)\left\|x_{n}-x^{*}\right\|^{2}+\alpha_{n}\left\|u-x^{*}\right\|^{2}-\left\|u_{n}-y_{n}\right\|^{2} .
\end{aligned}
$$

It follows that

$$
\left\|u_{n}-y_{n}\right\|^{2} \leq\left\|x_{n}-x^{*}\right\|^{2}-\left\|x_{n+1}-x^{*}\right\|^{2}+\alpha_{n}\left\|u-x^{*}\right\|^{2}
$$

Next, we consider two possible cases.

Case 1. Assume there exists some integer $m>0$ such that $\left\{\left\|x_{n}-x^{*}\right\|\right\}$ is decreasing for all $n \geq m$. In this case, we know that $\lim _{n \rightarrow \infty}\left\|x_{n}-x^{*}\right\|$ exists. From (3.19), we deduce

$$
\lim _{n \rightarrow \infty}\left\|u_{n}-y_{n}\right\|=0
$$

Returning to (3.17), we have

$$
\begin{aligned}
\left\|x_{n+1}-x^{*}\right\|^{2} \leq & \left\|y_{n}-x^{*}\right\|^{2} \\
\leq & \left(1-\alpha_{n}\right)\left\|x_{n}-x^{*}\right\|^{2}+\left(1-\alpha_{n}\right)\left(\delta^{2}\|A\|^{2}-\delta\right)\left\|S z_{n}-A x_{n}\right\|^{2} \\
& -\left(1-\alpha_{n}\right) \delta\left\|z_{n}-A x_{n}\right\|^{2}+\alpha_{n}\left\|u-x^{*}\right\|^{2} .
\end{aligned}
$$


Hence,

$$
\begin{aligned}
& \left(1-\alpha_{n}\right)\left(\delta-\delta^{2}\|A\|^{2}\right)\left\|S z_{n}-A x_{n}\right\|^{2}+\left(1-\alpha_{n}\right) \delta\left\|z_{n}-A x_{n}\right\|^{2} \\
& \leq\left\|x_{n}-x^{*}\right\|^{2}-\left\|x_{n+1}-x^{*}\right\|^{2}+\alpha_{n}\left\|u-x^{*}\right\|^{2}
\end{aligned}
$$

which implies that

$$
\lim _{n \rightarrow \infty}\left\|S z_{n}-A x_{n}\right\|=\lim _{n \rightarrow \infty}\left\|z_{n}-A x_{n}\right\|=0 .
$$

So,

$$
\lim _{n \rightarrow \infty}\left\|S z_{n}-z_{n}\right\|=0 .
$$

Note that

$$
\begin{aligned}
\left\|y_{n}-x_{n}\right\| & =\left\|\delta A^{*}\left(S P_{Q}-I\right) A x_{n}+\alpha_{n}\left(x_{n}-\delta A^{*}\left(I-S P_{Q}\right) A x_{n}-u\right)\right\| \\
& \leq \delta\|A\|\left\|S z_{n}-A x_{n}\right\|+\alpha_{n}\left\|x_{n}-\delta A^{*}\left(I-S P_{Q}\right) A x_{n}-u\right\| .
\end{aligned}
$$

It follows from (3.21) that

$$
\lim _{n \rightarrow \infty}\left\|x_{n}-y_{n}\right\|=0 \text {. }
$$

From (3.3), (3.12), and (3.17), we deduce

$$
\begin{gathered}
\left\|x_{n+1}-x^{*}\right\|^{2} \leq\left\|u_{n}-x^{*}\right\|^{2}-\beta_{n}\left(\gamma_{n}-\beta_{n}\right)\left\|u_{n}-T\left(\left(1-\gamma_{n}\right) u_{n}+\gamma_{n} T u_{n}\right)\right\|^{2} \\
\leq\left\|x_{n}-x^{*}\right\|^{2}+\alpha_{n}\left\|u-x^{*}\right\|^{2} \\
\quad-\beta_{n}\left(\gamma_{n}-\beta_{n}\right)\left\|u_{n}-T\left(\left(1-\gamma_{n}\right) u_{n}+\gamma_{n} T u_{n}\right)\right\|^{2} .
\end{gathered}
$$

It follows that

$$
\begin{aligned}
& \beta_{n}\left(\gamma_{n}-\beta_{n}\right)\left\|u_{n}-T\left(\left(1-\gamma_{n}\right) u_{n}+\gamma_{n} T u_{n}\right)\right\|^{2} \\
& \quad \leq\left\|x_{n}-x^{*}\right\|^{2}-\left\|x_{n+1}-x^{*}\right\|^{2}+\alpha_{n}\left\|u-x^{*}\right\|^{2} .
\end{aligned}
$$

Therefore,

$$
\lim _{n \rightarrow \infty}\left\|u_{n}-T\left(\left(1-\gamma_{n}\right) u_{n}+\gamma_{n} T u_{n}\right)\right\|=0 .
$$

Observe that

$$
\begin{aligned}
\left\|u_{n}-T u_{n}\right\| & \leq\left\|u_{n}-T\left(\left(1-\gamma_{n}\right) u_{n}+\gamma_{n} T u_{n}\right)\right\|+\left\|T\left(\left(1-\gamma_{n}\right) u_{n}+\gamma_{n} T u_{n}\right)-T u_{n}\right\| \\
& \leq\left\|u_{n}-T\left(\left(1-\gamma_{n}\right) u_{n}+\gamma_{n} T u_{n}\right)\right\|+L \gamma_{n}\left\|u_{n}-T u_{n}\right\|
\end{aligned}
$$

Thus,

$$
\left\|u_{n}-T u_{n}\right\| \leq \frac{1}{1-L \gamma_{n}}\left\|u_{n}-T\left(\left(1-\gamma_{n}\right) u_{n}+\gamma_{n} T u_{n}\right)\right\| .
$$


This together with (3.24) implies that

$$
\lim _{n \rightarrow \infty}\left\|u_{n}-T u_{n}\right\|=0
$$

Now, we show that

$$
\limsup _{n \rightarrow \infty}\left\langle u-x^{*}, y_{n}-x^{*}\right\rangle \leq 0
$$

Choose a subsequence $\left\{y_{n_{i}}\right\}$ of $\left\{y_{n}\right\}$ such that

$$
\limsup _{n \rightarrow \infty}\left\langle u-x^{*}, y_{n}-x^{*}\right\rangle=\lim _{i \rightarrow \infty}\left\langle u-x^{*}, y_{n_{i}}-x^{*}\right\rangle .
$$

Since the sequence $\left\{y_{n_{i}}\right\}$ is bounded, we can choose a subsequence $\left\{y_{n_{i j}}\right\}$ of $\left\{y_{n_{i}}\right\}$ such that $y_{n_{i j}} \rightarrow z$. For the sake of convenience, we assume (without loss of generality) that $y_{n_{i}} \rightarrow z$. Consequently, we derive from the above conclusions that

$$
x_{n_{i}} \rightarrow z, \quad u_{n_{i}} \rightarrow z, \quad A x_{n_{i}} \rightarrow A z \quad \text { and } \quad z_{n_{i}} \rightarrow A z .
$$

Applying Lemma 2.1, we deduce

$$
z \in \operatorname{Fix}(T) \text { and } A z \in \operatorname{Fix}(S) \text {. }
$$

Note that $u_{n_{i}}=P_{C} y_{n_{i}} \in C$ and $z_{n_{i}}=P_{Q} A x_{n_{i}} \in Q$. From (3.27), we deduce

$$
z \in C \text { and } A z \in Q \text {. }
$$

To this end, we deduce

$$
z \in C \cap \operatorname{Fix}(T) \text { and } A z \in Q \cap \operatorname{Fix}(S) .
$$

That is to say, $z \in \Gamma$.

Therefore,

$$
\begin{aligned}
\limsup _{n \rightarrow \infty}\left\langle u-x^{*}, y_{n}-x^{*}\right\rangle & =\lim _{i \rightarrow \infty}\left\langle u-x^{*}, y_{n_{i}}-x^{*}\right\rangle \\
& =\lim _{i \rightarrow \infty}\left\langle u-x^{*}, z-x^{*}\right\rangle \\
& \leq 0
\end{aligned}
$$

Using (2.5), we have

$$
\begin{aligned}
\left\|x_{n+1}-x^{*}\right\|^{2} & \leq\left\|y_{n}-x^{*}\right\|^{2} \\
& =\left\|\left(1-\alpha_{n}\right)\left(x_{n}-\delta A^{*}\left(I-S P_{Q}\right) A x_{n}-x^{*}\right)+\alpha_{n}\left(u-x^{*}\right)\right\|^{2} \\
& \leq\left(1-\alpha_{n}\right)\left\|x_{n}-\delta A^{*}\left(I-S P_{Q}\right) A x_{n}-x^{*}\right\|^{2}+2 \alpha_{n}\left\langle u-x^{*}, y_{n}-x^{*}\right\rangle \\
& \leq\left(1-\alpha_{n}\right)\left\|x_{n}-x^{*}\right\|^{2}+2 \alpha_{n}\left\langle u-x^{*}, y_{n}-x^{*}\right\rangle .
\end{aligned}
$$

Applying Lemma 2.2 and (3.28) to (3.29), we deduce $x_{n} \rightarrow x^{*}$. 
Case 2. Assume there exists an integer $n_{0}$ such that

$$
\left\|x_{n_{0}}-x^{*}\right\| \leq\left\|x_{n_{0}+1}-x^{*}\right\|
$$

Set $\omega_{n}=\left\{\left\|x_{n}-x^{*}\right\|\right\}$. Then we have

$$
\omega_{n_{0}} \leq \omega_{n_{0}+1}
$$

Define an integer sequence $\left\{\tau_{n}\right\}$ for all $n \geq n_{0}$ as follows:

$$
\tau(n)=\max \left\{l \in \mathbb{N} \mid n_{0} \leq l \leq n, \omega_{l} \leq \omega_{l+1}\right\} .
$$

It is clear that $\tau(n)$ is a non-decreasing sequence satisfying

$$
\lim _{n \rightarrow \infty} \tau(n)=\infty
$$

and

$$
\omega_{\tau(n)} \leq \omega_{\tau(n)+1}
$$

for all $n \geq n_{0}$.

By a similar argument to that of Case 1 , we can obtain

$$
\begin{aligned}
& \lim _{n \rightarrow \infty}\left\|u_{\tau(n)}-y_{\tau(n)}\right\|=\lim _{n \rightarrow \infty}\left\|x_{\tau(n)}-y_{\tau(n)}\right\|=0, \\
& \lim _{n \rightarrow \infty}\left\|S z_{\tau(n)}-A x_{\tau(n)}\right\|=\lim _{n \rightarrow \infty}\left\|z_{\tau(n)}-A x_{\tau(n)}\right\|=\lim _{n \rightarrow \infty}\left\|S z_{\tau(n)}-z_{\tau(n)}\right\|=0,
\end{aligned}
$$

and

$$
\lim _{n \rightarrow \infty}\left\|u_{\tau(n)}-T u_{\tau(n)}\right\|=0 .
$$

This implies that

$$
\omega_{w}\left(y_{\tau(n)}\right) \subset \Gamma
$$

Thus, we obtain

$$
\limsup _{n \rightarrow \infty}\left\langle u-x^{*}, y_{\tau(n)}-x^{*}\right\rangle \leq 0 .
$$

Since $\omega_{\tau(n)} \leq \omega_{\tau(n)+1}$, we have from (3.29) that

$$
\omega_{\tau(n)}^{2} \leq \omega_{\tau(n)+1}^{2} \leq\left(1-\alpha_{\tau(n)}\right) \omega_{\tau(n)}^{2}+2 \alpha_{\tau(n)}\left\langle u-x^{*}, y_{\tau(n)}-x^{*}\right\rangle .
$$

It follows that

$$
\omega_{\tau(n)}^{2} \leq 2\left\langle u-x^{*}, y_{\tau(n)}-x^{*}\right\rangle .
$$


Combining (3.30) and (3.32), we have

$$
\limsup _{n \rightarrow \infty} \omega_{\tau(n)} \leq 0
$$

and hence

$$
\lim _{n \rightarrow \infty} \omega_{\tau(n)}=0 .
$$

By (3.31), we obtain

$$
\limsup _{n \rightarrow \infty} \omega_{\tau(n)+1}^{2} \leq \limsup _{n \rightarrow \infty} \omega_{\tau(n)}^{2}
$$

This together with (3.33) implies that

$$
\lim _{n \rightarrow \infty} \omega_{\tau(n)+1}=0 .
$$

Applying Lemma 2.3 to get

$$
0 \leq \omega_{n} \leq \max \left\{\omega_{\tau(n)}, \omega_{\tau(n)+1}\right\}
$$

Therefore, $\omega_{n} \rightarrow 0$. That is, $x_{n} \rightarrow x^{*}$. This completes the proof.

Algorithm 3.3 For $x_{0} \in H_{1}$ arbitrarily, let $\left\{x_{n}\right\}$ be a sequence defined by

$$
\left\{\begin{array}{l}
u_{n}=P_{C}\left[\left(1-\alpha_{n}\right)\left(x_{n}-\delta A^{*}\left(I-S P_{Q}\right) A x_{n}\right)\right], \\
x_{n+1}=\left(1-\beta_{n}\right) u_{n}+\beta_{n} T\left(\left(1-\gamma_{n}\right) u_{n}+\gamma_{n} T u_{n}\right), \quad n \in \mathbb{N},
\end{array}\right.
$$

where $\left\{\alpha_{n}\right\}_{n \in \mathbb{N}},\left\{\beta_{n}\right\}_{n \in \mathbb{N}}$, and $\left\{\gamma_{n}\right\}_{n \in \mathbb{N}}$ are three real number sequences in $(0,1)$ and $\delta$ is a constant in $\left(0, \frac{1}{\|A\|^{2}}\right)$.

Corollary 3.4 Assume the following conditions are satisfied:

(C1) $\lim _{n \rightarrow \infty} \alpha_{n}=0$;

(C2) $\sum_{n=1}^{\infty} \alpha_{n}=\infty$;

(C3) $0<a<\beta_{n}<c<\gamma_{n}<b<\frac{1}{\sqrt{1+L^{2}}+1}$.

Then the sequence $\left\{x_{n}\right\}$ generated by algorithm (3.34) converges strongly to $x^{*}=P_{\Gamma}(0)$, which is the minimum norm in $\Gamma$.

Algorithm 3.5 For fixed $u \in H_{1}$ and $x_{0} \in H_{1}$ arbitrarily, let $\left\{x_{n}\right\}$ be a sequence defined by

$$
x_{n+1}=P_{C}\left[\alpha_{n} u+\left(1-\alpha_{n}\right)\left(x_{n}-\delta A^{*}\left(I-P_{Q}\right) A x_{n}\right)\right], \quad n \in \mathbb{N},
$$

where $\left\{\alpha_{n}\right\}_{n \in \mathbb{N}}$ is a real number sequence in $(0,1)$ and $\delta$ is a constant in $\left(0, \frac{1}{\|A\|^{2}}\right)$.

Corollary 3.6 Suppose $\Gamma_{1}$, the set of the solutions of (1.2), is nonempty. Assume the following conditions are satisfied: 
(C1) $\lim _{n \rightarrow \infty} \alpha_{n}=0$;

(C2) $\sum_{n=1}^{\infty} \alpha_{n}=\infty$.

Then the sequence $\left\{x_{n}\right\}$ generated by algorithm (3.35) converges strongly to $x^{*}=P_{\Gamma_{1}}(u)$.

Algorithm 3.7 For $x_{0} \in H_{1}$ arbitrarily, let $\left\{x_{n}\right\}$ be a sequence defined by

$$
x_{n+1}=P_{C}\left[\left(1-\alpha_{n}\right)\left(x_{n}-\delta A^{*}\left(I-P_{Q}\right) A x_{n}\right)\right], \quad n \in \mathbb{N}
$$

where $\left\{\alpha_{n}\right\}_{n \in \mathbb{N}}$ is a real number sequence in $(0,1)$ and $\delta$ is a constant in $\left(0, \frac{1}{\|A\|^{2}}\right)$.

Corollary 3.8 Suppose $\Gamma_{1}$, the set of the solutions of (1.2), is nonempty. Assume the following conditions are satisfied:

(C1) $\lim _{n \rightarrow \infty} \alpha_{n}=0$;

(C2) $\sum_{n=1}^{\infty} \alpha_{n}=\infty$.

Then the sequence $\left\{x_{n}\right\}$ generated by algorithm (3.36) converges strongly to $x^{*}=P_{\Gamma_{1}}(0)$, which is the minimum norm in $\Gamma_{1}$.

Algorithm 3.9 For fixed $u \in H_{1}$ and $x_{0} \in H_{1}$ arbitrarily, let $\left\{x_{n}\right\}$ be a sequence defined by

$$
\left\{\begin{array}{l}
u_{n}=\alpha_{n} u+\left(1-\alpha_{n}\right)\left(x_{n}-\delta A^{*}(I-S) A x_{n}\right), \\
x_{n+1}=\left(1-\beta_{n}\right) u_{n}+\beta_{n} T\left(\left(1-\gamma_{n}\right) u_{n}+\gamma_{n} T u_{n}\right), \quad n \in \mathbb{N},
\end{array}\right.
$$

where $\left\{\alpha_{n}\right\}_{n \in \mathbb{N}},\left\{\beta_{n}\right\}_{n \in \mathbb{N}}$, and $\left\{\gamma_{n}\right\}_{n \in \mathbb{N}}$ are three real number sequences in $(0,1)$ and $\delta$ is a constant in $\left(0, \frac{1}{\|A\|^{2}}\right)$.

Corollary 3.10 Suppose $\Gamma_{2}$, the set of the solutions of (1.3), is nonempty. Assume the following conditions are satisfied:

(C1) $\lim _{n \rightarrow \infty} \alpha_{n}=0$;

(C2) $\sum_{n=1}^{\infty} \alpha_{n}=\infty$;

(C3) $0<a<\beta_{n}<c<\gamma_{n}<b<\frac{1}{\sqrt{1+L^{2}}+1}$.

Then the sequence $\left\{x_{n}\right\}$ generated by algorithm (3.37) converges strongly to $x^{*}=P_{\Gamma_{2}}(u)$.

Algorithm 3.11 For and $x_{0} \in H_{1}$ arbitrarily, let $\left\{x_{n}\right\}$ be a sequence defined by

$$
\left\{\begin{array}{l}
u_{n}=\left(1-\alpha_{n}\right)\left(x_{n}-\delta A^{*}(I-S) A x_{n}\right), \\
x_{n+1}=\left(1-\beta_{n}\right) u_{n}+\beta_{n} T\left(\left(1-\gamma_{n}\right) u_{n}+\gamma_{n} T u_{n}\right), \quad n \in \mathbb{N},
\end{array}\right.
$$

where $\left\{\alpha_{n}\right\}_{n \in \mathbb{N}},\left\{\beta_{n}\right\}_{n \in \mathbb{N}}$, and $\left\{\gamma_{n}\right\}_{n \in \mathbb{N}}$ are three real number sequences in $(0,1)$ and $\delta$ is a constant in $\left(0, \frac{1}{\|A\|^{2}}\right)$.

Corollary 3.12 Suppose $\Gamma_{2}$, the set of the solutions of (1.3), is nonempty. Assume the following conditions are satisfied:

(C1) $\lim _{n \rightarrow \infty} \alpha_{n}=0$;

(C2) $\sum_{n=1}^{\infty} \alpha_{n}=\infty$;

(C3) $0<a<\beta_{n}<c<\gamma_{n}<b<\frac{1}{\sqrt{1+L^{2}}+1}$.

Then the sequence $\left\{x_{n}\right\}$ generated by algorithm (3.38) converges strongly to $x^{*}=P_{\Gamma_{2}}(0)$ which is the minimum norm in $\Gamma_{2}$. 
Example 3.13 Let $H_{1}=H_{2}=\mathbb{R}$ with the inner product defined by $\langle x, y\rangle=x y$ for all $x, y \in \mathbb{R}$ and the standard norm $|\cdot|$. Let $C=[0, \infty)$ and $Q=\mathbb{R}$. Let $S x=\frac{x}{2}-1$ for all $x \in Q$ and let $T x=x-1+\frac{4}{x+1}$ for all $x \in C$. Let $A x=-\frac{2 x}{3}$ for all $x \in \mathbb{R}$. Then $A$ is a bounded linear operator with its adjoint $A^{*}=A$. Observe that $\operatorname{Fix}(T)=3$ and $\operatorname{Fix}(S)=-2$. It is easy to see that

$$
\begin{aligned}
\langle T x-T y, x-y\rangle & =\left\langle x-1+\frac{4}{x+1}-y+1-\frac{4}{y+1}, x-y\right\rangle \\
& \leq\left[1-\frac{4}{(x+1)(y+1)}\right]|x-y|^{2} \\
& \leq|x-y|^{2},
\end{aligned}
$$

and

$$
\begin{aligned}
|T x-T y| & \leq\left|x-1+\frac{4}{x+1}-y+1-\frac{4}{y+1}\right| \\
& \leq\left|1-\frac{4}{(x+1)(y+1)}\right||x-y| \\
& \leq 5|x-y|,
\end{aligned}
$$

for all $x, y \in C$.

But

$$
\left|T\left(\frac{1}{4}\right)-T(0)\right|=\frac{11}{20}>\frac{1}{4} .
$$

Hence, $T$ is a Lipschitzian pseudocontractive mapping but not a nonexpansive one.

Note that $\|A\|=\left\|A^{*}\right\|=\frac{2}{3}$. Let $u=3$ and $\delta=\frac{9}{8}$. Then we have

$$
\begin{aligned}
u_{n} & =P_{C}\left[3 \alpha_{n}+\left(1-\alpha_{n}\right)\left(x_{n}-\frac{9}{8} \times\left(-\frac{2 I}{3}\right) \times\left(\frac{I}{2}+1\right) \times\left(\frac{-2 x_{n}}{3}\right)\right)\right] \\
& =P_{C}\left[3 \alpha_{n}+\left(1-\alpha_{n}\right)\left(\frac{3}{4} x_{n}+\frac{3}{4}\right)\right] .
\end{aligned}
$$

Let $\beta_{n}=\frac{1}{8}$ and $\gamma_{n}=\frac{1}{7}$ for all $n$. It is not hard to compute that

$$
\begin{aligned}
\left|x_{n+1}-3\right| & \leq\left|u_{n}-3\right| \\
& \leq\left(1-\alpha_{n}\right)\left|\frac{3}{4} x_{n}+\frac{3}{4}-3\right| \\
& \leq \frac{3}{4}\left|x_{n}-3\right| \\
& \leq \cdots \\
& \leq\left(\frac{3}{4}\right)^{n}\left|x_{1}-3\right|,
\end{aligned}
$$

which shows $x_{n} \rightarrow 3 \in \Gamma$. 


\section{Competing interests}

The authors declare that they have no competing interests.

\section{Authors' contributions}

All authors read and approved the final manuscript.

\section{Author details}

1 Department of Mathematics, Tianjin Polytechnic University, Tianjin, 300387, China. ${ }^{2}$ Department of Mathematics, Texas A\&M University, Kingsville, USA. ${ }^{3}$ Nonlinear Analysis and Applied Mathematics Research Group (NAAM), King Abdulaziz University, P.O. Box 80203, Jeddah, 21589, Saudi Arabia. ${ }^{4}$ Faculty of Applied Sciences, University 'Politehnica' of Bucharest, Splaiul Independentei 313, Bucharest, 060042, Romania. ${ }^{5}$ Department of Information Management, Cheng Shiu University, Kaohsiung, 833, Taiwan.

\section{Acknowledgements}

YY was supported in part by NSFC 71161001-G0105. Y-CL was supported in part by NSC 101-2628-E-230-001-MY3 and NSC 101-2622-E-230-005-CC3.

\section{Received: 11 April 2014 Accepted: 25 August 2014 Published: 02 Sep 2014}

\section{References}

1. Censor, Y, Elfving, T: A multiprojection algorithm using Bregman projections in a product space. Numer. Algorithms 8 , 221-239 (1994)

2. Censor, Y, Bortfeld, T, Martin, B, Trofimov, A: A unified approach for inversion problems in intensity modulated radiation therapy. Phys. Med. Biol. 51, 2353-2365 (2006)

3. Censor, Y, Elfving, T, Kopf, N, Bortfeld, T: The multiple-sets split feasibility problem and its applications for inverse problems. Inverse Probl. 21, 2071-2084 (2005)

4. Censor, Y, Motova, A, Segal, A: Perturbed projections and subgradient projections for the multiple-sets split feasibility problem. J. Math. Anal. Appl. 327, 1244-1256 (2007)

5. Byrne, C: Iterative oblique projection onto convex subsets and the split feasibility problem. Inverse Probl. 18, 441-453 (2002)

6. Xu, HK: Iterative methods for the split feasibility problem in infinite-dimensional Hilbert spaces. Inverse Probl. 26, 105018 (2010)

7. Byrne, $\mathrm{C}$ : A unified treatment of some iterative algorithms in signal processing and image reconstruction. Inverse Probl. 20, 103-120 (2004)

8. Censor, Y, Segal, A: The split common fixed point problem for directed operators. J. Convex Anal. 16, 587-600 (2009)

9. Yu, X, Shahzad, N, Yao, Y: Implicit and explicit algorithms for solving the split feasibility problem. Optim. Lett. (2012). doi:10.1007/s11590-011-0340-0

10. Qu, B, Xiu, N: A note on the CQ algorithm for the split feasibility problem. Inverse Probl. 21, 1655-1665 (2005)

11. Zhao, J, Yang, Q: Several solution methods for the split feasibility problem. Inverse Probl. 21, 1791-1799 (2005)

12. Dang, Y, Gao, Y: The strong convergence of a KM-CQ-like algorithm for a split feasibility problem. Inverse Probl. 27, 015007 (2011)

13. Wang, F, Xu, HK: Approximating curve and strong convergence of the $C Q$ algorithm for the split feasibility problem. J. Inequal. Appl. (2010). doi:10.1155/2010/102085

14. Wang, Z, Yang, Q, Yang, Y: The relaxed inexact projection methods for the split feasibility problem. Appl. Math. Comput. (2010). doi:10.1016/j.amc.2010.11.058

15. Yao, Y, Wu, J, Liou, YC: Regularized methods for the split feasibility problem. Abstr. Appl. Anal. 2012, Article ID 140679 (2012)

16. Yao, Y, Postolache, M, Liou, YC: Strong convergence of a self-adaptive method for the split feasibility problem. Fixed Point Theory Appl. 2013, Article ID 201 (2013)

17. Moudafi, A: The split common fixed-point problem for demicontractive mappings. Inverse Probl. 26, 055007 (2010)

18. Wang, F, Xu, HK: Cyclic algorithms for split feasibility problems in Hilbert spaces. Nonlinear Anal. 74, 4105-4111 (2011)

19. Zhao, J, He, S: Alternating Mann iterative algorithms for the split common fixed-point problem of quasi-nonexpansive mappings. Fixed Point Theory Appl. 2013, Article ID 288 (2013)

20. Zhao, J, He, S: Simultaneous iterative algorithms for the split common fixed-point problem of generalized asymptotically quasi-nonexpansive mappings without prior knowledge of operator norms. Fixed Point Theory Appl. 2014, Article ID 73 (2014)

21. Chang, SS, Kim, J, Cho, YJ, Sim, J: Weak and strong convergence theorems of solutions to split feasibility problem for nonspreading type mapping in Hilbert spaces. Fixed Point Theory Appl. 2014, Article ID 11 (2014)

22. Cui, H, Wang, F: Iterative methods for the split common fixed point problem in Hilbert spaces. Fixed Point Theory Appl. 2014, Article ID 78 (2014)

23. Zhou, $\mathrm{H}$ : Strong convergence of an explicit iterative algorithm for continuous pseudocontractions in Banach spaces. Nonlinear Anal. 70, 4039-4046 (2009)

24. Xu, HK: Iterative algorithms for nonlinear operators. J. Lond. Math. Soc. 66, 240-256 (2002)

25. Mainge, PE: Approximation methods for common fixed points of nonexpansive mappings in Hilbert spaces. J. Math. Anal. Appl. 325, 469-479 (2007) 